\title{
Representations of Graph Temperley-Lieb Algebras
}

By

Paul P. Martin*

\begin{abstract}
We define graph Temperley-Lieb algebras, which are certain generalisations of Temperley-Lieb algebras including those appropriate for building the transfer matrices for lattice models in arbitrary dimensions. We construct various representations for these algebras, which are parameterised by a scalar $Q$. In particular we give the generically irreducible representations associated with statistical mechanical models. These representations are well defined for all $Q$, whereas the representations coming directly from physical models are typically only defined for certain values of $Q$. We also give representations with bases derived from the partitions of $n$ distinguishable objects ( $n$ is the number of nodes in the graph). We show how to compute the dimensions of these representations by a diagrammatic technique.
\end{abstract}

\section{\$1. Introduction}

There has been much diverse interest recently in Temperley-Lieb $(T L)$ algebras, $T_{n}(Q)[1,2,3,4]$, which may be defined as follows. Associate a generator $U_{i}$ with each node $i$ of an $A_{n}$ graph (Figure 1). The generators have relations

$$
\begin{aligned}
U_{i} U_{i} & =\sqrt{ } Q U_{i} \\
U_{i} U_{j} U_{i} & =U_{i} \cdots \text { if nodes } i \text { and } j \text { are connected by a bond, } \\
U_{i} U_{j} & =U_{j} U_{i} \cdots \text { otherwise. }
\end{aligned}
$$

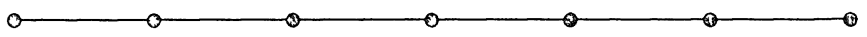

Figure 1: The $A_{n}$ graph for $n=7$

The physical motivation for the study of these algebras comes from the algebraic formulation of the transfer matrix for certain $2 \mathrm{~d}$ statistical mechanical models including the $Q$-state Potts model (see, for example, [2]).

In the present paper we consider the effect of generalising the graph associated with the defining relations (1) to any graph constructed as

Communicated by H. Araki, June 10, 1989. Revised July 31, 1989.

* Department of Mathematics, City University, Northampton Square, London ECIV OHB U. K. 
follows. Form a 'pregraph' from a collection of nodes or 'sites' somehow connected by bonds, the graph is obtained by decorating the middle of every bond with a further site ${ }^{1}$. The algebras associated with the relations (1) in such cases will be called graph Temperley-Lieb $(G T L)$ algebras. The algebra associated with a given pregraph $G$ will be denoted $G T(Q)$ (so $T_{2 n-1}(Q)$ becomes $A_{n} T(Q)$ and so on). We examine the representation theory for these new algebras. The immediate application of this work is in the $3 \mathrm{~d}$ Potts spin and lattice gauge models, for which the algebra generators for an appropriate graph build the $2 \mathrm{~d}$ layer transfer matrix. The identification of the irreducible representation associated with the free energy in these models vastly simplifies the computation of the free energy on the finite lattice. The breakdown of the $Q$-generic structure of the algebra should signal a $3 \mathrm{~d}$ series of critical field theory limits analogous to the central charge $c \leq 1$ conformal series $\left(Q=4 \cos ^{2}(\pi / r)\right.$; $r \in Z, r \neq 0$ ) in $2 \mathrm{~d}$.

In the next section we give the representations of graph Temperley-Lieb algebras (with $Q$ positive integer) associated with Potts models, and describe the quotient relations obeyed by these representations (a proof of this result is outlined in appendix $\mathrm{A} 1$, while the irreducible content of these representations is discussed in A2). In section 3 we give representations (with $Q$ an indeterminate) associated with the set of partitions of $n$ distinguishable objects (the order of this set is given in appendix A3). We identify generically irreducible subspaces and determine the corresponding quotient relations. In section 4 we discuss the physical consequences of these results.

\section{§2. The Potts Representation}

\subsection{Construction}

For definiteness, then, let us start by considering the algebras appropriate for the $3 \mathrm{~d}$ Potts models. The pregraphs here are square lattices, so the graphs are edge centred square lattices. The $3 \mathrm{~d}$ Potts $l . m$-site layer transfer matrix may be written

$$
T(v)=\prod_{\text {layersites } i .}\left(v+Q^{1 / 2} U_{i .}\right) \prod_{\text {layer bonds } i j}\left(1+v Q^{-1 / 2} U_{i j}\right)
$$

where $v=(\exp (\beta)-1)$ and $\beta$ is the coupling parameter, as in the $2 \mathrm{~d}$ case [2]. The layer site label (i.) may be regarded as a vector specifying a site position in the layer $i .=\left(i_{1}, i_{2}\right)\left(i_{1} \in 1, \ldots, l ; i_{2} \in 1, \ldots, m\right)$; and the bond label $(i j)$ as a pair of vectors specifying adjacent (ie. bond connected) sites in the layer.

\footnotetext{
${ }^{1}$ There are many further generalisations possible, involving directed bonds, multiple bonds and so on. Our choice is motivated by physical considerations (see later) given which such further complications are as yet superfluous.
} 
For more general pregraphs the site label is not naturally specified as a $2 \mathrm{~d}$ vector. We will, however, retain the distinction of indexing pregraph sites with a dotted letter, and bonds with the associated pair of sites. This pregraph notation seems to be more convenient in general than labelling generators with a node of the derived graph (as is done in the relations (1)). Henceforward we will always specify algebras by their pregraph, and the relations (1) (which apply to the graph) should be interpretted accordingly.

The matrices $U_{i}$, and $U_{i j}$ are given as follows:

$$
U_{i .}, U_{i j} \in \operatorname{End}\left(\bigotimes_{i_{1}=1, \ldots, l ; i_{2}=1, \ldots, m} V_{i .}\right)
$$

where $V_{i}$ is the $Q$ dimensional vector space with basis the possible Potts spin variable values on site $(i$.$) , say s_{i .} \in\{1, \ldots, Q\}$. Then:

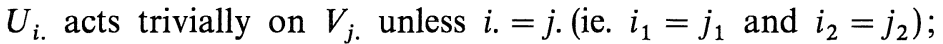

$U_{i \text {. on }} V_{i}$ is $Q^{-1 / 2} M$, where $M$ is the $Q$-by- $Q$ matrix with all entries unity.

Meanwhile:

$U_{i j}$ acts trivially on $V_{k}$. unless $i .=k$. or $j .=k$;

$U_{i j}$ on $V_{i .} \otimes V_{j .}$ is diagonal $\left(Q^{1 / 2} \delta\left(s_{i .}, s_{j .}\right)\right)$.

Note that the generalisation of this definition to arbitrary graphs is automatic.

\subsection{The Potts quotient algebra}

We may think of these matrices as defining a finite dimensional 'Potts' algebra for each integer $Q$ (the dimension of such an algebra is $\leq Q^{l m}$ ) or we may think of them as giving a representation of the abstract algebra associated with the original graph. The quotient relations describing the place of the Potts algebra in the abstract algebra are not obvious, but note, for instance, that the Potts representation is real and symmetric, while representations need not be unitarisable in general. The abstract algebra can be finite dimensional only if the pregraph has no closed loops and no nodes with coordination number greater than 3. We will give a further discussion of this result elsewhere.

In any case the Potts representation must obey at least one set of quotient relation on physical grounds, if they are not already a consequence of the relations (1). That is, the transfer matrix $T(0)$ should be a primitive idempotent (at least up to normalisation). For the $A_{n} T(Q)$ algebras this is already a consequence of the original relations, but in other cases it simply amounts to the statement that at high temperatures the Potts model is disordered. The explicit relations may be determined by reference to inhomogeneous Potts model partition functions with free boundary conditions in the layering direction.

By similar arguments a minimal list of sets of quotient relations appropriate 
for such physical models (and all of which are trivial in the $A_{n}$ case) is indicated as follows. For $W$ any word of length $O(W)$ in the generators $\left\{U_{i,}, U_{i j}\right\}$ and

$$
R_{b^{(N)}}=\prod_{i . \notin b^{(N)}}\left(U_{i .} / Q^{1 / 2}\right)
$$

where $b^{(N)}$ is any $N$ element subset of nodes in the pregraph, so $0 \leq N \leq n$, and, for example,

$$
R_{0}=R_{b^{(0)}}=\prod_{\text {all pregraph nodes } i .}\left(U_{i .} / Q^{1 / 2}\right)
$$

then:

$$
R_{b^{(N)}} W R_{b^{(N)}}=\chi_{b^{(N)}}(W) R_{b^{(N)}} \quad\left(\bmod . R_{b^{(M)}} \forall b^{(M)} \subset b^{(N)}\right)
$$

where $\chi_{b^{(N)}}(W)$ is a scalar, and in particular, generically $\chi_{b^{(n)}}(W)=0(O(W)$ $>0$ ). The proof is outlined in the appendix A1.

This means that we may, in principle, associate not necessarily distinct irreducible representations with the left ideals generated from each $R_{b^{(N)}}\left(\bmod . R_{b^{(M)}}\right)$.

\section{§3. Partition Representations}

\subsection{The bases}

Note that for $Q=0$ the Potts representation is undefined, and for $Q=1$ it is 1 dimensional $\left(U_{i .}=U_{i j}=1 \forall i, j\right)$. Otherwise the representations grow rapidly with $l, m$ and it is possible to show directly that they are reducible (see appendix A2). Furthermore, it is not obvious how to generalise to non-integer $Q$, although the defining relations make no obvious distinction. Let us proceed by constructing 2 further types of representation from a different (although related) source. One of these types is generically irreducible and both are defined for all $Q$. The idea for these representations comes from the Whitney polynomial [2] for certain non-planar graphs which we will describe later. However, the basis is most naturally described in a more general framework.

Consider the set of partitions of $n$ distinguishable objects. For example with $n=1,2,3$ we have $\{(1)\},\{(1)(2),(12)\},\{(1)(2)(3),(12)(3),(13)(2),(23)(1)$, (123)\}. Another useful notation here is to replace the list of all partitions in each case with just the list of all partition shapes (as in Young diagrams [9]) preceded by their corresponding multiplicities (if other than unity). In this scheme the $n=3$ set becomes $\left\{\left(1^{3}\right), 3 .(21),(3)\right\}$. We will define $\mathscr{S}_{n}$ to be the number of partitions in each case. Then for $n=1,2,3,4,5,6,7,8$ we have $\mathscr{S}_{n}$ $=1,2,5,15,52,203,877,4140$, and so on. We will show how to compute these 
numbers in the appendix A3.

If 2 objects ( $a, b$ say) are clustered together in a partition we say that they are connected, and write $a \sim b$. Of course $a \sim b$ and $b \sim c$ implies $a \sim c$.

Now associate to each partition the set of possible partitions of its connected clusters into 2 'teams' (including the possibility of empty teams). If a partition has $c$ clusters then the set has $2^{c}$ elements. For example the set for $(13)(2)$ is $\{((13)(2), \emptyset),((13),(2)),((2),(13)),(\emptyset,(13)(2))\}$. Here $((13)(2), \emptyset)$ means the element in which the clusters (13) and (2) are in the first team, and the second team is empty. The union of such sets over the partitions then provides a basis for a representation of any $G T L$ algebra with an $n$ node pregraph, as we will see shortly.

It is convenient to partially order the union by putting all the elements with $i$ cluster ' $2^{\text {nd }}$ teams' before all those with $j$ cluster $2^{\text {nd }}$ teams if $i<j$; and within this ordering by putting all elements with $d$ clusters in total before those with $e$ clusters in total if $d>e$. The number of elements in the union with $i$ cluster $2^{\text {nd }}$ teams, $\mathscr{S}_{n}(i)$, is computed in the appendix. Note that we have put the $\left(1^{n}\right)$ partitions first in each fixed $i$ subset.

\subsection{Representation type 1}

Assign one distinguishable object (from $n$ ) to each site of the pregraph. Then numbering elements in the union from $1, \ldots, \mathscr{S}_{n}(0), \mathscr{S}_{n}(0)+1, \ldots, \mathscr{S}_{n}(0)$ $+\mathscr{S}_{n}(1), \ldots, \sum_{i} \mathscr{S}_{n}(i)$ the representation is given by

$$
\begin{aligned}
&\left(U_{i .}\right)_{k l}= Q^{\delta_{k l} / 2} \ldots \text { if completely disconnecting the object } \\
& \text { at site } i . \text { of the pregraph and putting it in } \\
& \text { the } 1^{\text {st }} \text { team takes element } k \text { to } l, \\
&=0 \ldots \text { otherwise; } \\
&\left(U_{i j}\right)_{k l}= Q^{\delta_{k l} / 2} \ldots \text { if connecting the objects } \\
& \text { at sites } i \text {. and } j . \text { of the pregraph, and putting } \\
& \text { the resultant cluster in the } 2^{n d} \text { team if } i \text { and or } \\
& j \text { is in the second team, takes element } k \text { to } l, \\
&=0 \ldots \text { otherwise. }
\end{aligned}
$$

To see that this defines a representation note (cf. the defining relations):

i) that the repeated application of $U_{i}$. or $U_{i j}$ simply results in the appearance of a factor of $Q^{1 / 2}$;

ii) that the order of application of any 2 operators is unimportant if they are not of the form $U_{i}$. and $U_{j k}$ with either $i .=j$. or $i .=k$;

iii) that disconnecting an object, connecting it and then disconnecting it again is equivalent to disconnecting it;

and iv) that connecting 2 objects, disconnecting one and then reconnecting it is equivalent to connecting the 2 objects. QED. 
Note that no operation increases the number of clusters in the second team, so we have a sequence of invariant subspaces filtered by this number. Quotienting by elements with second team occupancy $<i$ we get representations for each fixed $i$. We call the fixed $i$ basis the 'full' partition basis for each $i$.

Note that all these bases depend on the pregraph only in as much as they depend on $n$.

\subsection{Representation type 2}

The above representations are not, in general, irreducible. Fixing $i$, there is an invariant subspace associated with the sub-basis of elements with the property that they can be realised as 'boundary states' of walks on an extended pregraph (e.p.graph) constructed as follows (see also Figure 2).
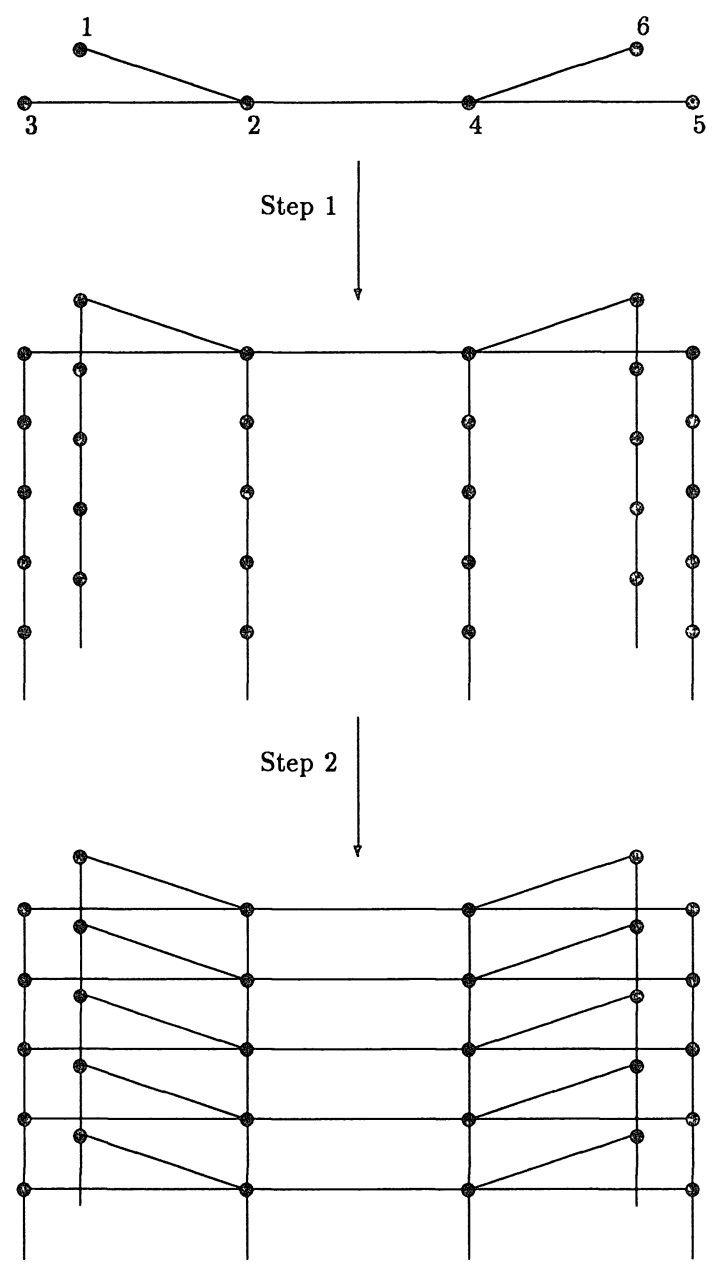

Figure 2: Constructing the e.p. graph for the pregraph $D_{5}^{(1)}$ 
Step (1): To each node of the pregraph associate an infinite tower of nodes connected by a linear chain of bonds. The original node is the top level node of the tower (call it level 0).

Step (2): Considering the original pregraph, join the nodes at each given level in the resultant set of towers in the same way as the original nodes at level 0 are joined to construct the pregraph.

Now consider again the set of partitions of $n$ distinguishable objects into clusters, with $i$ clusters in the second team. The subset of elements we want for an invariant subspace is that set for which it is possible to construct nonintersecting paths along bonds of the extended pregraph such that each set of connected nodes in the partition at level 0 is joined by a path, and that each cluster in the second team is joined by a path to a level with all nodes otherwise pathless.

To see this note from the definition (7) and the subsequent quotienting procedure that each $U_{i}$. or $U_{i j}$ has at most one non-zero entry per column. Thus starting from any basis state corresponding to all nodes disconnected, and acting with some $U_{i}$. or $U_{i j}$, the effect is just to take us to another (or possibly the same) basis state. The new state is obtained from the old one by either connecting 2 nodes (acting with $U_{i j}$ ) or disconnecting one $\left(U_{i .}\right)$. Any word in the $U$-operators ( $W$, say) takes us from the original basis state to another by a sequence of moves corresponding to connecting nodes or disconnecting a node. Writing the disconnected basis state as $|0\rangle$ we can express the effect of the sequence of moves by

$$
W\left|0>=k_{W}\right| w>,
$$

where $|w\rangle$ is the basis state reached by the action of $W$ and $k_{W}$ is some scalar function of $Q^{1 / 2}$ (see below). Note that we have distinguished between the labels $W$ and $w$ since, in general, more than one word $W$ will produce a given state $\mid w>$.

Now each of the moves in turn may be represented diagramatically by adding a 'layer' (consisting of new level 0 pregraph and bonds connecting each node to the corresponding node in the next level) to the top of the e.p.graph. The layer must contain appropriate sections of paths, or 'steps', on some bonds. If a path is present between two nodes then they are connected, otherwise they are disconnected. Thus the required layer for a $U_{i}$. contains steps on every bond between levels except at node $i$, and no paths between nodes within a level; while the layer for a $U_{i j}$ contains steps on every bond between levels and a step between nodes $i$ and $j$ within a level. The effect of a $U_{i}$ or $U_{i j}$ on the present state depends only on the resultant connectivity of nodes at level 0 (although in general this connectivity will be achieved by paths passing through other levels in the e.p.graph), so any paths or parts of paths irrelevant to this connectivity, such as cul-de-sacs, may be ignored. This is why we have 


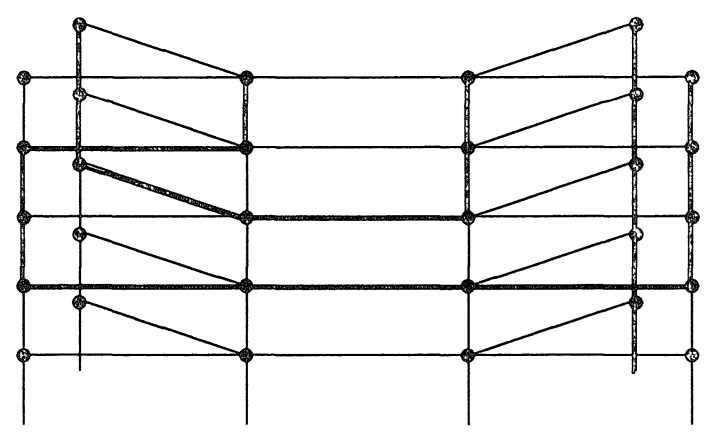

Figure 3: Realisation of the basis state $((14)(25)(3),(6))$ for the pregraph $D_{5}^{(1)}$. Note that node 6 at level 0 is connected to the corresponding node in the bottom level shown, which is otherwise totally disconnected.

described this realisation of basis states as 'boundary states' of walks on the e.p.graph.

We note immediately that, for an arbitrary pregraph, not all connectivities can be realised in this way, thus not all basis states are in the invariant subspace. We can determine the invariant subspace by carrying out a programme of adding layers with steps to the e.p.graph with no paths (i.e. acting with $U_{i}$ and $U_{i j}$ on some totally disconnected basis state) and noting the accessible connectivities, until the set of connectivities so produced becomes fixed.

For example, in the case shown in figure 2 (i.e. for $n=6$ with the pregraph $\left.D_{5}^{(1)}\right)$, the basis state $((14)(25)(36), \emptyset)$ is not accessible, since any connection between 1 and 4 leaves room for only one non-intersecting path from 2,3 to 5,6, while we require two. By the same measure $((14)(25)(3)(6), \emptyset)$ is possible. Similarly, in the case $i=1$ the state $((14)(25)(6),(3))$ is not accessible, while the state $((14)(25)(3),(6))$ is accessible. An appropriate arrangement of paths in this case is shown by the thick lines in Figure 3 (note that since we need only keep information about the connectivity of the top level it is often possible, and convenient, to compose the effects of several layers as defined above into one layer, which may then isolate or connect several nodes at once).

Carrying out the programme indicated above for the $D_{5}^{(1)}$ pregraph shown here we find that 198 out of $203 i=0$ basis states are accessible. In fact we have computed the $i=0$ accessible subspace for many pregraphs by this straightforward procedure. But the results show no simple patterns as yet to suggest a more sophisticated algorithm in general. We will discuss this problem further elsewhere.

For another example, if the pregraph is $A_{4}$ then the extended pregraph is a 4 site wide square lattice. Numbering the nodes of the pregraph (at level 0 on the e.p.graph) from one end to the other we see that we have excluded the basis 
state $((13)(24), \emptyset)$ from the subspace, since there is no path on the extended pregraph from 1 to 3 which does not cross any path from 2 to 4 . Similarly $((13)(4),(2))$ is excluded, since any path from 1 to 3 isolates 2 from all pathless levels.

In general the invariant subspaces correspond to the left ideals generated from $R_{b^{(\imath)}}\left(\bmod R_{b^{(\jmath)}} b^{(j)} \subset b^{(i)}\right)$ in the quotient algebra in which $R_{b^{(j)}}\left(\forall b^{(j)} ; j\right.$ $=0, \ldots, i)$ is a primitive idempotent, as follows. The basis element with all sites isolated, and nodes in $b^{(i)}$ in the second team, corresponds to $R_{b^{(i)}}$. Multiplying (on the left) by any pre-graph bond $U_{i j}$ which does not change the second team occupancy gives the partition with the appropriate pair of sites connected, and so on. This process just corresponds to reproducing the abovementioned paths at the operator level. An example of an excluded element, at second team occupancy $i=2$ in the $A_{4}$ case, is $U_{12} U_{3} U_{4}$, since $U_{1} U_{3} U_{4}=0\left(\bmod R_{b^{(1)}}, b^{(1)}\right.$ $=\{i .=2\}$ ) implies $U_{12} U_{1} U_{3} U_{4} U_{12}=0$.

For completeness we note that, up to overall factors of $Q^{1 / 2}$, the element of the left ideal generated from $U_{1} U_{2} U_{3} U_{4} U_{5}$ represented in figure 3 may be written

$$
U_{3} U_{23} U_{2} U_{12} U_{24}\left(U_{1}\right) U_{2} U_{4} U_{23} U_{24} U_{45}\left(U_{1} U_{2} U_{3} U_{4} U_{5}\right) U_{1} U_{2} U_{3} U_{4} U_{5} .
$$

This product, taken from left to right, can be extracted from the figure by reading from top to bottom. The bracketed factors each reduce to $Q^{1 / 2}$ on application of the defining relations (from eqn(1)).

In general if we replace every factor of the form $U_{i}$. by $\left(U_{i .} Q^{1 / 2}\right)$ and every factor of the form $U_{i j}$ by $\left(U_{i j} Q^{-1 / 2}\right)$ in a word $W$ then the factor $k_{W}\left(Q^{1 / 2}\right)$ in $W \mid 0>$ $=k_{W}\left(Q^{1 / 2}\right) \mid w>$ becomes just $Q^{C}$. Here $C$ is the number of isolated paths, including single nodes, in the interior of the e.p.graph (i.e. those which do not affect the level 0 connectivity). In our example, for instance, the above renormalisation changes each of the bracketed factors to $Q$, corresponding to the presence of the isolated node in position 1 at level 3 (that is the fourth level down) and the isolated nodes in positions $1,2,3,4$ and 5 at level 4 (the bottom level shown). We will prove the general result in section 3.6.

In the corresponding representations $T^{k}(v)$ is, up to similarity transformations, the transfer matrix for the dichromatic polynomial [2] $(Q$ is the other parameter) associated with an extended pregraph of just $k$ levels. This is, perhaps, a strange object to consider. Nonetheless, it provided the stimulation for these ideas! The extended pregraph of $k$ levels is a useful notion for later on. We will adopt the notation $G^{k}$ for such an extension of the graph $G$.

\subsection{The fixed $n$ pregraph dependence of type 2 bases}

Note, from the generalised version of the definition (1), that if an algebra has a pregraph $G$, and another algebra has a pregraph $H$, then $G T(Q)$ is a subalgebra of $H T(Q)$ if $H$ contains $G$ as a subgraph. The 'maximal' pregraph with $n$ nodes 
is the one in which every site is connected to every other by a bond. We sometimes call this the infinite dimensional case, in reference to the associated physical models. At the other extreme, a graph with more than one connected component has an algebra corresponding to the direct product of commuting algebras associated with each connected component.

In the case $i=0$, note that the algebras for the pregraphs in which at least one site is connected by a bond to every other have an irreducible representation given by the full partition basis $(i=0)$, since in these cases every partition may be realised from the $\left(1^{n}\right)$ partition by some sequence of moves in which sites are connected along bonds or sites are isolated. The instances in which such pregraphs are also tree graphs are unique for each $n$ (up to permutations of sites), having one coordination number $(n-1)$ site and $(n-1)$ coordination number one sites. These cases are called 'daisy' graphs.

For general $i$ only the algebra for the maximal pregraph always has an irreducible representation associated with the full fixed $i$ basis.

As bonds are removed from the maximal pregraph, then, the subspaces accessed from $\left(1^{n}\right)$ (ie. the $\left(1^{n}\right)$ elements in any fixed $i$ basis) in the way described above occasionally shrink. One possible limit of this procedure is the case in which all site coordination numbers are 2 (except for a pair of coordination number 1 sites at the ends of the chain of bonds). We sometimes call this $A_{n}$ case the 'linear' pregraph. This case is the original $T L$ algebra for which such a sub-basis was discussed in Martin 1986 [6]. Here the number of accessible partitions is $C_{n}=1,2,5,14,42,132, \ldots$ with

$$
\lim _{n \rightarrow \infty} C_{n+1} / C_{n}=4
$$

$[7]^{2}$. Of course the linear pregraph is not the unique connected endpoint of such a bond dissolution process (but that's another story). The assymptotic growth rate above tells us that every positive integer $Q$ value below 4 has an exceptional (ie. non-generic) $T L$ algebra associated for large enough $n$, as we will see shortly.

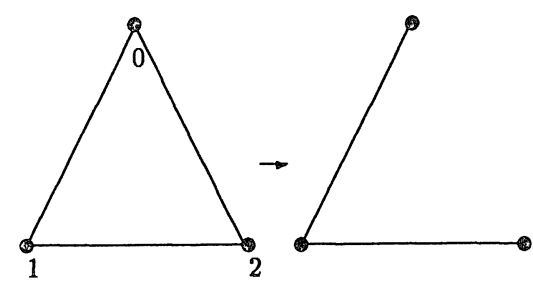

Figure 4: The maximal $\left(A_{2}^{(1)}\right)$ and daisy/linear $\left(A_{3}\right)$ pregraphs for $n=3$

\footnotetext{
${ }^{2}$ The number of accessible basis elements for linear pregraphs and general $i$ is, by continuity [8], the dimension of the irreducible representation of the symmetric group $S_{2 n}$ corresponding to the 2 row tableau shape $(n+i, n-i)$.
} 


\subsection{Generic irreducibility of type 2 representations}

First let us show that $R_{b^{(l)}}$ is, at least up to quotients, a primitive idempotent, and hence that (provided the algebra is semi-simple) the representation associated with the left ideal is irreducible (otherwise it is merely indecomposable).

For given $n$, consider the tree of pregraphs related by bond dissolutions starting from the maximal case and ending with tree graphs (which become disconnected if any further bonds are dissolved). For $n=1,2$ the endpoints coincide. For $n=3$ we have just 2 possible connected graphs, Figure 4 . In both cases the $i=0$ irreducible subspace coincides with the original 5 dimensional space, but for $A_{2}^{(1)}$ we must impose the non-trivial quotient relation

$$
U_{0} U_{1} U_{2} U_{01} U_{12} U_{20} U_{0} U_{1} U_{2}=y\left(Q^{1 / 2}\right) U_{0} U_{1} U_{2}
$$

(where $y$ is known - see later) on the abstract algebra (1) before $R_{b^{(0)}}=Q^{-3 / 2} U_{0}$ $U_{1} U_{2}$ becomes primitive.

For $n=5$ the daisy graph endpoint is shown in Figure 5. For the corresponding algebra $R_{b^{(\jmath)}}$ is a primitive idempotent modulo quotient relations of the form

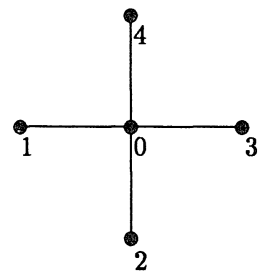

Figure 5: The daisy pregraph $\left(D_{4}^{(1)}\right)$ for $n=5$

$$
R_{b^{(l)}} U_{01} U_{03} U_{0 .} U_{02} U_{04} U_{0 .} U_{01} U_{03} U_{0 .} U_{02} U_{04} R_{b^{(l)}}=x\left(Q^{1 / 2}\right) R_{b^{(l)}}
$$

To determine the list of such relations in general is difficult and, in fact, unnecessary.

To see that $R_{b^{(l)}}$ is primitive (modulo such relations) in general, ie. for any graph, note that all the $U_{j}$. are lower triangular in any representation with fixed second team occupancy $i$, so that $\left(R_{b^{(i)}}\right)_{k k}$ is zero unless all the $\left(U_{j}\right)_{k k}\left(j . \notin b^{(i)}\right)$ are non-zero. Each $\left(U_{j}\right)_{k k}$ is non-zero only if disconnecting a site leaves the basis element $k$ alone, so for any partition other than of $\left(1^{n}\right)$ type some such matrix element must be zero. Similarly, for $\left(1^{n}\right)$ type elements of the form $\left(\left(m_{1}\right)\left(m_{2}\right) \ldots\right.$ $\left.\left(m_{n-i}\right),\left(m_{n-i+1}\right) \ldots\left(m_{n}\right)\right)$ where any $m_{p>n-i} \notin b^{(i)}$, the action of $R_{b^{(i)}}$ is to reduce the number of second team clusters. Conversely for the unique $\left(1^{n}\right)$ type element of the form $\left(\left(m_{1}\right)\left(m_{2}\right) \ldots\left(m_{n-i}\right),\left(m_{n-i+1}\right) \ldots\left(m_{n}\right)\right)$ where $b^{(i)}=\left\{m_{n-i+1}, \ldots, m_{n}\right\}$ (num- 
bered $k=1$, say), no $\left(U_{j}\right)_{11}\left(j . \notin b^{(i)}\right)$ is zero. QED. Alternatively, note that the action of $R_{b^{(2)}}$ on any element of the fixed second team occupancy $i$ subset is either to take it to "zero" (ie. an element of occupancy $<i$ ) or to take it to the element $k=1$ above, since $R_{b^{(i)}}$ disconnects and puts into the first team all nodes $\notin b^{(i)}$. Thus either the result has less than $i$ clusters in the second team or it is precisely $k=1$.

This argument also allows a straightforward determination of the required quotient relations in each case, eg. the form of $x\left(Q^{1 / 2}\right)$ in equation (10).

The representations on the accessible subspaces for any pregraph are thus irreducible provided the algebra is semi-simple.

\subsection{Quotient relations for type 2 representations}

It follows from the treatment of the Potts representation in appendix A1, and in particular the e.p.graph representation of words $W(Q)$, together with the discussion in section 3.3, that the quotient relations generically coincide (when $Q \in Z_{+}$, and formally regarding $Q$ as an indeterminate integer in A1).

To see this note the following. Forming the word $W^{\prime}(Q)$ (where $W^{\prime}$ $=R_{b^{(M)}} W R_{\left.b^{(M)}\right)}$ in the e.p.graph, it describes some connectivity from one end of the e.p.graph to the other, and some connectivity of nodes at each end of the e.p.graph, together with some isolated clusters. From the definition of type 2 representations we will show that here, as in the Potts case, either the number of distinct lines passing through the diagram decreases or $\chi_{b^{(M)}}(W)$ (defined by analogy with eqn(6)) just picks up a factor of $Q$ for each isolated cluster.

Specifically, note that all the $U_{i}$. matrices in eqn(7) may be arranged to be lower triangular; and the $U_{i j}$ matrices upper triangular. There is, therefore, an equivalent representation (for $Q \neq 0$ ) with the non-vanishing matrix elements in eqn(7) replaced by

$$
\left(U_{i .}\right)_{k l}=Q^{1 / 2} \text { and }\left(U_{i j}\right)_{k l}=Q^{\delta_{k l}-1 / 2}
$$

Recall that the quotient relations are basis independent. Then note that:

(i) introducing $U_{i j} Q^{-1 / 2}$ into $W(Q)$ cannot change the number of isolated clusters if it does not change the connectivity; but if it does change the connectivity then it must do so by connecting 2 isolated clusters, and so reduce the number of isolated clusters by 1 . Meanwhile, in our equivalent representation, the appropriate matrix element is such as to introduce a factor of precisely 1 in the former situation and $Q^{-1}$ in the latter.

(ii) On the other hand, $U_{i} . Q^{1 / 2}$ increases the number of isolated clusters by 1 whether or not it changes the connectivity (since either way it isolates a node). But from the definition of the equivalent representation the matrix element of $U_{i .} Q^{1 / 2}$ is precisely $Q$ in either situation. 
This result implies that the Potts representation is, generically, just a direct sum of type 2 representations. We have not determined the multiplicities in general.

\section{§4. Physical Consequences}

We can show that the type 2 representation associated with $R_{0}$ is the representation associated with the free energy in statistical mechanical models. To see this note that the free energy is the largest magnitude eigenvalue of the transfer matrix when the coupling is real. In this case the transfer matrix is positive, ie. all the elements are positive, and by Perron's theorem it has a unique largest magnitude eigenvalue, which is positive and has a 'positivisable' (ie. positive up to an overall phase) eigenvector. From the definition it is easy to see that $R_{0}$ is the matrix with all entries 1 (up to an overall factor) in the Potts representation. Since it is a primitive idempotent this implies that the Potts representation contains the irreducible representation discussed above exactly once as an irreducible component (and also as an indecomposable module). Again from the Potts representation it is easy to see that $R_{0}$ is not orthogonal to the positivisable eigenvector associated with the free energy in this basis. Therefore the irreducible representation associated with the primitive idempotent $R_{0}$ is the one responsible for the part of the transfer matrix spectrum containing the free energy.

Note that the Potts representation has asymptotic growth rate of dimension $d_{n}(n$ sites $)$ of

$$
\lim _{n \rightarrow \infty} d_{n+1} / d_{n}=Q
$$

This is independent of the graph. If we take linear pregraphs then the irreducible from $R_{0}$, contained in the Potts representation, has generic asymptotic growth rate 4 (see above). This means that for large enough $n$ and $Q<4$ the generic 'irreducible' representation contained in the Potts representation has dimension greater than the Potts representation. In such cases the true irreducible must be smaller than the generic irreducible, ie. the left ideal generated from the primitive idempotent is no longer the basis for an irreducible representation. This means that the abstract algebra is no longer semisimple. In other words the $T L$ algebra has an exceptional structure for $Q$ $=0,1,2,3$ (with $Q=4$ a limiting case of the generic structure). This observation has been the subject of much interest in $2 \mathrm{~d}$ physics in the last couple of years or so [5].

Asymptotic growth rates for arbitrary sequences of pregraphs are not known at present. The most interesting question for physics is perhaps the asymptotic growth rate for pregraphs associated with $3 \mathrm{~d}$ models. The rate for 
coordination number $n$ pregraphs (ie. essentially infinite dimensional models) is infinite. This means that here all integer $Q$ values are exceptional for large enough $n$. The proof is given in the appendix. Work is in progress to determine the $3 \mathrm{~d}$ asymptotics.

I would like to thank the RIMS team for many stimulating discussions. The recollection of past discussions with $\mathrm{H} \mathrm{N} \mathrm{V} \mathrm{Temperley} \mathrm{and} \mathrm{B}$ W Westbury has made my job much easier, and I would also like to thank my colleagues at the University of Birmingham for granting me study leave so that I might develop these ideas in Kyoto.

\section{Appendix}

\section{A.1 Quotient relations for the Potts representation}

We outline a demonstration that the Potts representation obeys the quotient relations (6).

$(N=0)$ In the Potts representation $\left(R_{0}\right)_{i j}=Q^{-n} \forall i, j$. QED.

$(N=1) R_{b^{(1)}}$ takes the form $M_{n-1} \otimes 1_{Q}$ where $M_{n-1}$ is $R_{0}$ for a graph with the node $b^{(1)}$ deleted and $1_{Q}$ is the $Q$ dimensional unit matrix. But the Potts generators are all invariant under a global redefinition of site variables so, with $E_{i j}$ a $Q$ dimensional elementary matrix,

$$
R_{b^{(1)}} W R_{b^{(1)}}=\sum_{i, j=1, \ldots, Q} C_{W}(i, j) M_{n-1} \otimes E_{i j}
$$

where $C_{W}(i, i)$ is independent of $i$ and $C_{W}(i, j) i \neq j$ is independent of $i$ and $j$. Now quotient by $R_{0}$ from the $(N=0)$ case above. QED.

$(N>1)$ It is useful to define an inhomogeneous transfer matrix, generalising equation (2), by

$$
T\left(\left\{v_{i .}, v_{i j}\right\}\right)=
$$

$\prod_{\text {pregraph nodes } i .}\left(\left(v_{i .}+Q^{1 / 2} U_{i .}\right) /\left(1+v_{i .}\right)\right) \prod_{\text {pregraph bonds } i j}\left(\left(1+v_{i j} Q^{-1 / 2} U_{i j}\right) /\left(1+v_{i j}\right)\right)$

Clearly any word $W$ can then be written as a product of such transfer matrices, since any generator can. The physical picture is of a Potts system with some couplings at zero temperature $(\beta=\infty, v=\infty)$ so that the connected spins are frozen together; and some couplings at high temperature $(\beta=0, v=0)$ so that the connected spins are effectively decoupled. We see from the transfer matrix that whether or not a generator is included in each case depends on whether it is associated with a node or a bond. From the definition of the Potts generators we confirm that the presence of a bond generator freezes the relevant spins together, while a node generator decouples them. It is then helpful to write out $W$ on $G^{k}{ }^{3}(k$ as large as necessary to contain $W$, ie. $k \leq O(W))$. The 
nodes of $G^{k}$ can be thought of as the sites of a Potts lattice. The transfer matrix transfers attention from one level to the next. If a bond is frozen in the transfer matrix then it is marked in the e.p.graph.

For example, a marked e.p.graph for

$$
\begin{aligned}
W= & U_{1 .} U_{3 .} U_{5 .} U_{6 .} U_{12} U_{23}\left(U_{56}\right) U_{54} U_{5 .} U_{4 .} U_{54} \\
& U_{56} U_{5 .} U_{6 .} U_{56} U_{3 .} U_{1 .} U_{34} U_{21} U_{2 .} U_{4 .} U_{34} U_{21} U_{1 .} U_{3 .} U_{5 .} U_{6 .}
\end{aligned}
$$

in $A_{6} T(Q)$ is shown in Figure 6. The construction is precisely analogous to that described for the e.p.graph path realisation of basis states for partition representations in section 3.3, with path 'steps' replaced by marked bonds. Note, however, that the e.p.graph in figure 6 has been drawn on its side, compared to figure 2. In figure 6 , in analogy to figure 3 , we have, where possible, accounted for more than one factor in a layer - in general any number of successive factors of the form $U_{i}$. followed by any number of successive factors of the form $U_{i j}$ may clearly be incorporated in the same layer. In the diagram each $U_{i}$. (ie. unmarked horizontal bond) should be read as $Q^{1 / 2} U_{i}$. and each $U_{i j}$ (marked vertical bond) as $Q^{-1 / 2} U_{i j}$ (from equation (13)). We will denote by $W(Q)$ the product obtained from $W$ by making these replacements.

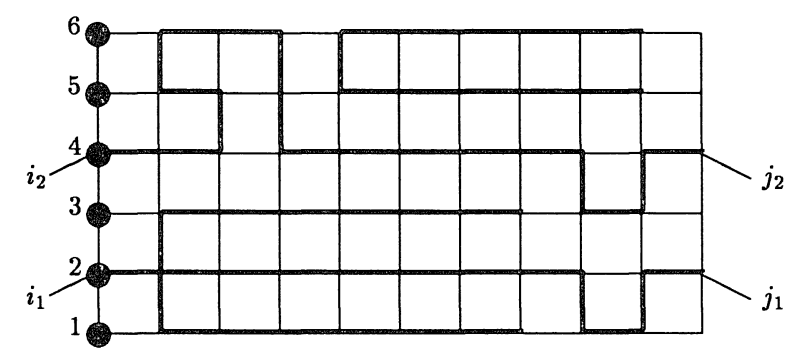

Figure 6: A word from $A_{6} T(Q)$ drawn on $\left(A_{6}\right)^{11 .}$

Any $W$ can be represented this way, and any such diagram corresponds to some $W$. In general the word may be reducible (ie. $W \propto W^{\prime}$ with $O(W)$ $\left.>O\left(W^{\prime}\right)\right)$ by the relations (1). In our example $W(Q)=Q W^{\prime}(Q)$ where $W^{\prime}$ $=U_{1} U_{3} U_{5} U_{6}$. . The factor of $Q$ comes from the relations (1), but physically it corresponds to the fact that the isolated cluster in the top right of the diagram may take any of $Q$ possible values for its spins (which are frozen together). The remaining spins are determined by the boundary conditions. For general pregraphs the word will not necessarily reduce using the relations in this way, but isolated clusters will clearly still give rise to factors of $Q$, from the physical

\footnotetext{
${ }^{3}$ The extended pregraph (e.p.graph) $G^{k}$ is defined in section 3.3.
} 
picture, in the Potts representation.

Now $R_{b^{(N)}}$ takes the form $M_{n-N} \otimes 1_{Q^{N}}$, so

$$
R_{b^{(N)}} W R_{b^{(N)}}=\sum_{i_{1}, j_{1}, \ldots, i_{N}, j_{N}=1, \ldots, Q} C_{W}\left(i_{1}, j_{1}, \ldots, i_{N}, j_{N}\right) M_{n-N} \otimes E_{i_{1}, j_{1}} \otimes \ldots \otimes E_{i_{N}, j_{N}}
$$

This corresponds to imposing free boundary conditions at the 'external' points $i_{a}, j_{a} \notin b^{(N)}$ (in the pregraphs at levels 0 and $(k-1)$ of the e. p. graph, respectively) and then specifying completely the boundary states of $i_{a}, j_{a} \in b^{(N)}$ to obtain a matrix element $C_{W}$. By writing the whole of the left hand side as a word (for example, in the figure we have $R_{b^{(2)}} W^{\prime} R_{b^{(2)}}$ where $\left.b^{(2)}=\{2,4\}\right)$ we see that, for large enough $Q, C_{W}$ just depends, up to overall factors, on the connectivity of the 'external' nodes $i_{a}$ and $j_{a}\left(i_{a}, j_{a} \in b^{(N)}\right)$ of $G^{k}$. If the boundary conditions are consistent with the connectivity (ie. if $i_{a} \sim j_{b}$ then the spins $i_{a}=j_{b}$; if $i_{a} \sim i_{b}$ then $i_{a}=i_{b}$; and if $j_{a} \sim j_{b}$ then $j_{a}=j_{b}$ ) the result is just $Q^{c}$ where $c$ is the number of isolated connected clusters. Otherwise it is zero. We thus see that, up to overall factors, any $R_{b^{(N)}} W R_{b^{(N)}}$ diagram may be contracted to its 'basic' connections before computing $C_{W}$. Because of the $R_{b(N)}$ factors at each end, the only case not covered by the quotienting is $i_{a} \sim j_{b}$ if and only if $a=b$. All other connectivities involve fewer distinct (non-interconnected) lines passing through the diagram, and can thus be represented by elements of ideals already quotiented out (recall equation (6)).

To see this in an example, consider the case in which $W$ from eqn(14) is replaced by the same thing but with the bracketed factor $\left(U_{56}\right)$ missing. Call this word $X$. Then only one line passes through the diagram, and $i_{2} \nsucc j_{2}$. In this case the relations simplify the word to $Q^{(n-1) / 2} R_{b^{(1)}}$ with $b^{(1)}=\{2\}$. Specifically, $X(Q)=Q Q^{(n-1)} R_{b^{(1)}}$. Again the frozen bond picture ensures that such a simplification occurs on physical grounds in the Pottts representation, even if the relations (1) do not imply it.

In general, if $i$ distinct lines cross the diagram, then the corresponding word is in the double sided ideal generated from some $R_{b^{(i)}}$ (since adding generators to a word never increases the number of distinct lines across its diagram). Note that all cases in which $i_{a}=j_{a}$ are covered by the quotient relations or by $R_{b(N)}$, and all cases in which $i_{a} \neq j_{a}$ for some $i_{a} \in b^{(N)}$ are covered by the quotient relations. That is, such boundary conditions imply a maximum amount of connectedness across the diagram.

We have shown that the left hand side of eqn(15) can be replaced by the left hand side of eqn (6).

For example, with $N=2$, if all 4 external nodes are disconnected then the $L H S$ of eqn (15) is in the double sided ideal generated from $R_{0}$. If all 4 are connected then it is in the ideal generated from $R_{b^{(1)}}$, and so on.

Finally, note that for sufficiently small $Q$ there are more connectivities 
possible than states of the boundary nodes, so the connectivities cannot be treated independently. We will discuss the consequences of this limitation elsewhere, but basically it is a signal of the breakdown of the generic structure of the algebra.

\section{A.2 Reducibility of the Potts representation}

To see that the Potts representation for $Q=2,3,4, \ldots$ is reducible consider the similarity transformation by the matrix $S$ defined by

$$
\begin{aligned}
S_{i j} & =(1 /(Q+1-i)) \quad \cdots j \geq i \\
S_{i i-1} & =-1 \\
S_{i j} & =0 \ldots \text { otherwise. }
\end{aligned}
$$

on every space $V_{k .}$. The site operators then become diagonal $\left(Q^{1 / 2}, 0,0, \ldots\right)$ and the bond operators do not mix between states with odd and even numbers of spins in state $s_{k}=Q$. We will discuss the further reducibility of the Potts representation for $Q>2$ elsewhere.

We know from the $A_{n}$ case that the decomposition described here for $Q=2$ is complete. This is a remarkable result. It means that the $Q=2 A_{n}$ Potts algebra is isomorphic to every other $Q=2 n$-node pregraph Potts algebra. In other words the operators for building arbitrary dimensional interactions may be built from just one local operator and a set of spatially translating and rotating conjugations, all of which already exist in the $A_{n}$ (ie. $2 \mathrm{~d}$ Potts model) algebra.

For $Q=3$ we have shown that the Potts representation contains at least 2 irreducible components. On the other hand we know from the $A_{n}$ case that there are at most 3. We also know on physical grounds that the multiplicities of the first 2 irreducibles are 1 and 2 respectively. The lower bounds for their dimensions (and that of the third, if it exists) from the $A_{n}$ case, together with the total dimension of the Potts representation and the dimensions of the blocks we exhibited above, are given in the following table for enough cases to show the pattern:

$\begin{array}{rrrrrrr}n & d 1 & d 2 & d 3 & d \text { Potts } & b 1 & b 2 \\ 1 & 1 & 1 & 0 & 3 & 2 & 1 \\ 2 & 2 & 3 & 1 & 9 & 5 & 4 \\ 3 & 5 & 9 & 4 & 27 & 14 & 13 \\ 4 & 14 & 27 & 13 & 81 & 41 & 40\end{array}$

We see that the only possibility is that, here also, the $A_{n}$ Potts algebra is isomorphic to every other $n$-node pregraph algebra. The same remarkable consequences also pertain.

In fact the same is trivially true for $Q=0,1$. On the other hand it is not true for $Q$ large enough. We will discuss these results further elsewhere. 


\section{A.3 Computation of $\mathscr{S}_{n}(i)$}

To compute $\mathscr{S}_{n}(0)$ note that it is the sum of entries in the $n^{\text {th }}$ row of the following diagram:

$\begin{array}{rrrrrr}1 & & & & & \\ 1 & 1 & & & & \\ 1 & 3 & 1 & & & \\ 1 & 7 & 6 & 1 & & \\ 1 & 15 & 25 & 10 & 1 & \\ . . & . . & . & . . & . . & . .\end{array}$

where the number in the $j^{\text {th }}$ column of the $n^{\text {th }}$ row is the sum of the number in the $(j-1)^{t h}$ column of the $(n-1)^{t h}$ row and $j$ times the number in the $j^{\text {th }}$ column of the $(n-1)^{t h}$ row. To see this note that $\mathscr{S}_{n}(0)$ is also the number of sequences of length $n, \mathbf{p}=\left\{p_{1}, p_{2}, \ldots, p_{n}\right\}$, with the property that

$$
1 \leq p_{i} \leq\left(\left(\max p_{j} \forall 1 \leq j \leq i\right)+1\right) .
$$

Proof. Associate $p_{i}$ with node $i$ and say nodes $i, j$ are connected if $p_{i}=p_{j}$ (ie. label the relevant cluster by $p_{i}$ ). Then the cluster containing node 1 is always cluster no. 1 , that containing node 2 is cluster no. 2 unless it is in cluster no. 1 , and so on. It is easy to see that this is a unique representation of partitions.

On the other hand the possibilities for $\mathbf{p}$ may be enumerated diagrammatically as follows:

$$
\begin{aligned}
& \mathrm{P}_{1}: \quad 1 \\
& \mathrm{P}_{2}: \quad 12 \\
& \begin{array}{lll|llll}
\mathrm{P}_{3}: & 1 & 2 & 1 & 2 & 3
\end{array} \\
& \mathrm{P}_{4}: 12 \text { | } 11223 \mid \begin{array}{lll|lll|llll}
1 & 2 & 3 & 1 & 2 & 3 & 1 & 2 & 3 & 4
\end{array}
\end{aligned}
$$

and so on. The claimed result follows almost immediately.

Note also from this construction that the $j^{\text {th }}$ number in the $n^{\text {th }}$ row in the original diagram gives the number of partitions into exactly $j$ non-empty clusters. Note that the maximal such contribution to any row comes from further to the right as $n$ increases, so the asymptotic ratio is

$$
\lim _{n \rightarrow \infty} \mathscr{S}_{n+1} / \mathscr{S}_{n}=\infty
$$

To compute $\mathscr{S}_{n}(i)$ note that the number of ways of choosing $i$ clusters from $j$ to go into the second team is $(j) ! /(j-i) !(i) ! j \geq i$ and zero otherwise. Thus the corresponding diagram for arbitrary $i$ is obtained by multiplying each entry in the diagram above by the appropriate factor. For example for $i=1,2,3$ we have: 


$\begin{array}{rrrrr}1 & & & & \\ 1 & 2 & & & \\ 1 & 6 & 3 & & \\ 1 & 14 & 18 & 4 & \\ 1 & 30 & 75 & 40 & 5 \\ 0 & & & & \\ 0 & 1 & & & \\ 0 & 3 & 3 & & \\ 0 & 7 & 18 & 6 & \\ 0 & 15 & 75 & 60 & 10 \\ 0 & & & & \\ 0 & 0 & & & \\ 0 & 0 & 1 & & \\ 0 & 0 & 6 & 4 & \\ 0 & 0 & 25 & 40 & 10\end{array}$

\section{References}

[1] Temperley, H. N. V. and E. Lieb, Proc. R. Soc., 332 (1971), 251.

[2] Baxter, R. J., Exactly Solved Models in Statistical Mechanics, Academic, New York, 1982.

[3] Pasquier, V., Nucl. Phys., B285 (1987), 162; Date, E., M. Jimbo, A. Kuniba, T. Miwa and M. Okado, Nucl. Phys., B290(FS20) (1987), 231.

[4] Kuniba, A., Y. Akutsu and M. Wadati, J. Phys. Soc. Japan, 55 (1986), 3285; Deguchi, T., M. Wadati and Y. Akutsu, J. Phys. Soc. Japan, 57 (1988), 1905.

[5] Cardy, J., Conformal Field Theory, in Phase Transitions and Critical Phenomena eds. Domb and Lebowitz, vol. 11, Academic, New York, 1986.

[6] P. P. Martin, J. Phys., A19 (1986), L1117.

[7] Blote, H. W. J. and M. Nightingale, Physica, 112A (1982), 405.

[8] P. Hoefsmit, Representations of Hecke Algebras..., University of British Columbia thesis, 1974.

[9] Robinson, G. de B., Representation Theory of the Symmetric Group, University of Toronto Press, Toronto, 1961. 
